\title{
Entorno laboral saludable en las instituciones educativas y riesgos psicosociales del personal docente
}

\section{Healthy work environment in educational institutions and psychosocial risks of teaching staff}

\author{
Carlos Javier Spencer Silva ${ }^{1 *}$, Marianela Silva Sánchez ${ }^{1}$, María Caridad Mederos Machado ${ }^{1}$ y Marllelis \\ Gutiérrez Hinestroza ${ }^{1}$ \\ ${ }^{1}$ Universidad Estatal Península de Santa Elena \\ *huvillospencer@gmail.com
}

DOI: https://doi.org/10.26871/killkana_social.v2i4.371

\begin{abstract}
Resumen
El presente artículo tiene como objetivo determinar la importancia de un entorno laboral saludable en las instituciones de educación para la minimización de los riesgos psicosociales del personal docente. En el mismo se desarrollan conceptualizaciones generales sobre las dos variables y se clasifican los riesgos psicosociales, para lo cual se analiza las propuestas conceptuales de la Organización Mundial de la Salud (2010), Organización Panamericana de la Salud (2015) y la Organización Internacional del Trabajo. Se usó como metodología la revisión bibliográfica de los documentos oficiales que al respecto publican estas tres organizaciones e investigaciones relacionadas la temática como las de García (2016) y Moreno y Báez (2010) entre otras. Se destacan entre las consideraciones finales la coincidencia que existe entre los tres organismos al manifestar que para alcanzar entornos laborales saludables, se deben evitar al máximo los riesgos psicosociales a través de la promoción de ambientes sanos, mejorando de esta manera la calidad de vida de los docentes. La correcta adecuación de todos los ámbitos que confluyen en un mismo espacio de trabajo es fundamental para conseguir un entorno laboral favorable que prevenga riesgos y fomente la salud y el bienestar entre los docentes. De igual manera resalta la importancia, que cada día se afianza con mayor fuerza, de consolidar entornos laborales saludables, donde los riesgos psicosociales se muestran como factores de indiscutible estudio y búsqueda sostenida y efectiva de soluciones prácticas con énfasis en el logro del bienestar físico y mental del talento humano.
\end{abstract}

Palabras clave: entorno laboral saludable, riesgos psicosociales, personal docente, instituciones educativas.

\begin{abstract}
The objective of this article is to determine the importance of a healthy work environment in educational institutions for minimizing the psychosocial risks of teaching staff. In the same general conceptualizations are developed on the two variables and psychosocial risks are classified, for which conceptual proposals of the World Health Organization (2010), Pan American Health Organization (2015) and the International Organization of the Work. The bibliographic review of the official documents published by these three organizations and research related to the topic such as García (2016) and Moreno and Báez. (2010), among others, was used as a methodology. Among the final considerations are the coincidence that exists between the three organizations when they state that in order to achieve healthy working environments, psychosocial risks should be avoided to the maximum through the promotion of healthy environments, thus improving the quality of life of the teachers. The correct adaptation of all areas that come together in the same work space is essential to achieve a favorable work environment that prevents risks and promotes health and well-being among teachers. It also highlights the importance, which is becoming stronger every day, of consolidating healthy work environments, where psychosocial risks are shown as factors of unquestionable study and sustained and effective search for practical solutions with emphasis on the achievement of physical well-being and mental of human talent.
\end{abstract}

Key words: ealthy work environment, psychosocial risks, professor, educational institutions.

\section{Introducción}

En el presente artículo se realiza una revisión documental sobre el entorno laboral saludable en las instituciones educativas y los riesgos psicosociales del docente, con la finalidad de determinar su importancia para el lo- gro de una mejor salud ocupacional como factor positivo en el desarrollo socioeconómico y calidad de vida de la población, además de motivar la formulación y cumplimiento de políticas necesarias que consideren los avances $\mathrm{y}$ actualizaciones referidas a los nuevas tendencias del 
quehacer educativo, como trabajo de medio tiempo, trabajos a distancia, trabajo en casa, el hogar como escuela, trabajos que involucran desplazamientos y autoempleo, y las bases ocupacionales que han sido creadas en respuesta a esta diversidad laboral escolar ajustada a las exigencias educacionales mundiales.

\section{Entorno laboral saludable}

\subsection{Definiciones Generales}

La Organización Panamericana de la Salud (2015) considera al lugar de trabajo como un entorno prioritario para la promoción de la salud en el siglo XXI. La salud en el trabajo y los entornos laborales saludables se cuentan entre los bienes más preciados de personas, comunidades y países. Un entorno laboral saludable es esencial, no sólo para lograr la salud de los trabajadores en general y los docentes en particular, sino también para hacer un aporte positivo a la productividad, la motivación laboral, el espíritu de trabajo, la satisfacción en el trabajo y la calidad de vida general. Un lugar de trabajo saludable promueve una buena salud, que es un recurso primordial para el desarrollo social, económico y personal, así como una importante dimensión de los ambientes laborales.

Las definiciones de entorno de trabajo saludable han evolucionado enormemente durante las últimas décadas. Desde un casi exclusivo enfoque sobre el ambiente físico de trabajo (en el esquema tradicional de la salud y seguridad ocupacionales, que consideran los riesgos físicos, químicos, biológicos y ergonómicos), la definición se ha ampliado hasta incluir hábitos de salud (estilos de vida); factores psicosociales (organización del trabajo y cultura de trabajo); y establecer nexos con la comunidad, y todo lo que pueda tener un profundo efecto en la salud del empleado. (Organización Mundial de la Salud, 2010)

Basado en lo establecido en el documento de la Organización Mundial de la Salud (2010) se asume como concepto de entorno laboral saludable al estado de completo bienestar físico, mental y social, y no la simple ausencia de la enfermedad. A este concepto se ajustan las definiciones que al respecto hacen diferentes instituciones u organizaciones a nivel mundial. Es así como La Oficina Regional del Pacífico Oeste de la OMS específica que un entorno de trabajo saludable, es "un lugar donde todos trabajan unidos para alcanzar un visión conjunta de salud y bienestar para los trabajadores y la comunidad. Esto proporciona a todos los miembros de la fuerza de trabajo, condiciones físicas, psicológicas, sociales y organizacionales que protegen y promueven la salud y la seguridad". (Organización Mundial de la Salud, 2010, p.14)

En tanto Grawitch y otros (2009, citados por OMS, 2010) consideran que la clave para un entorno de trabajo saludable está en la introducción de intervenciones efectivas para el balance vida-trabajo. Mientras que para Pease (2006), Messing y Östlin (2006, citados por OMS, 2010) un tema que se retoma en muchos artículos y publicaciones sobre entornos laborales saludables, es el concepto de inclusividad o diversidad, donde el entorno de trabajo saludable debe proporcionar un ambiente abierto de accesibilidad y aceptación para personas de diferente sustrato, origen, capacidades y habilidades. También debe asegurar que se minimicen o eliminen las disparidades entre grupos de trabajadores o las dificultades que afecten a grupos específicos.

Con base a lo anteriormente expuesto, se considera que un entorno de trabajo saludable es aquel en el que los trabajadores y jefes colaboran en un proceso de mejora continua para promover y proteger la salud, seguridad y bienestar de los trabajadores y la sustentabilidad del ambiente de trabajo en base a los siguientes indicadores: (a) La salud y la seguridad concernientes al espacio físico de trabajo. (b) La salud, la seguridad y el bienestar concernientes al medio psicosocial del trabajo incluyendo la organización del mismo y la cultura del ambiente de trabajo. (c) Los recursos de salud personales en el espacio de trabajo, y (d) Las formas en que la comunidad busca mejorar la salud de los trabajadores, sus familias y de otros miembros de la comunidad. (Organización Mundial de la Salud, 2010)

Derivado de los diferentes conceptos presentados, se puede afirmar que diversos factores como los políticos, económicos, sociales, culturales, ambientales y biológicos pueden favorecer o dañar la salud de los docentes, constituyéndose en sí mismo en un proceso político y social que abarca no solamente las acciones dirigidas a fortalecer las habilidades y capacidades de los individuos, sino también las dirigidas a modificar las condiciones que en las diversas áreas favorezcan de la productividad y competitividad de las instituciones educativas y contribuyendo al desarrollo económico y social de los países.

\section{Riesgos psicosociales}

Las cambiantes condiciones económicas y sociales de nuestra sociedad repercuten en una evolución constante de los lugares de trabajo. Esta situación está conduciendo a la aparición de nuevos riesgos vinculados al modo en que se organiza el trabajo en las instituciones educativas y a las relaciones socio laborales frente a los que es necesario actuar. Factores como el nivel de atención requerido para el desarrollo de una actividad, el ritmo y carga de trabajo, la comunicación ineficaz o la monotonía de la tarea, entre otros, pueden contribuir a desencadenar accidentes de trabajo y enfermedades profesionales. (Zimmermann, 2016)

Como complemento a lo expuesto, Bezanilla y otros (2018) expresan que la psicología ha identificado tres elementos fundamentales que conforman a la persona: biológico, psicológico, social. El componente biológico se relaciona con todos aquellos aspectos relacionados con la fisiología y anatomía del cuerpo. El componente psicológico se encuentra integrado por los procesos de pensamiento, senso-percepción, motivación, emoción, integrados en una estructura de personalidad que conforma la unidad psicológica del individuo. El aspecto social de la persona tiene que ver con los distintos roles que representa, los grupos a los 
que pertenece, el tipo de relaciones sociales que establece. Estos tres componentes cuando interactúan conforman un sistema que debe estar en equilibrio para que se considere que la persona se encuentra en óptimo estado de salud.

Existen distintos factores que pueden poner en riesgo el equilibrio biopsicosocial de la persona, trayendo como consecuencia la presencia de distintas enfermedades, ya sean en el cuerpo (cáncer, diabetes, gripa, sida, hipertensión), el estado psicológico (trastornos del estado de ánimo, trastornos de ansiedad, trastornos somatoformes, trastornos fácticos, trastornos disociativos) o la capacidad de adaptación social de la persona ( bajo rendimiento escolar, consumo de sustancias, conductas antisociales, aislamiento, falta de adaptación, entre otros)

\subsection{Definición de riesgo psicosocial}

Moreno y Báez (2010) realizan una revisión bibliográfica (Carayon, Haims y Yang (2001), Cox y Griffiths (1996), Martín Daza y Pérez Bilbao (1997) que deriva en la condensación de elementos que conforman la definición de riesgo psicosocial: características percibidas del ambiente de trabajo que tienen una connotación emocional para los docentes y directivos; aspectos del trabajo, de la organización y de la gestión laboral, y sus contextos sociales y organizacionales; condiciones que se encuentran presentes en una situación laboral que están directamente relacionadas con la organización, el contenido del trabajo y la realización de la tarea, y que tienen capacidad para afectar tanto al bienestar o a la salud (física, psíquica o social) del docente como al desarrollo de su trabajo.

Por su parte la Organización Internacional del Trabajo (1986), citado por Moreno y Báez,(2010) define los factores psicosociales en el trabajo como las interacciones entre el trabajo, su medio ambiente, la satisfacción en el trabajo y las condiciones de organización, por una parte, y por la otra, las capacidades del trabajador, sus necesidades, su cultura y su situación personal fuera del trabajo, todo lo cual, a través de percepciones y experiencias, puede influir en la salud, en el rendimiento y en la satisfacción en el trabajo.

En relación al tema, la Agencia Europea para la Seguridad y Salud en el Trabajo (2018) expresa que los riesgos psicosociales se derivan de las deficiencias en el diseño, la organización y la gestión del trabajo, así como de un escaso contexto social del trabajo, y pueden producir resultados psicológicos, físicos y sociales negativos, como el estrés laboral, el agotamiento o la depresión. Algunos ejemplos de condiciones de trabajo que entrañan riesgos psicosociales son: cargas de trabajo excesivas; exigencias contradictorias y falta de claridad de las funciones del puesto; falta de participación en la toma de decisiones que afectan al docente; inseguridad en el empleo; comunicación ineficaz, falta de apoyo por parte de la dirección o los compañeros; acoso psicológico y sexual.

En el mismo orden de ideas, riesgo psicosociales, es una terminología que refiere a hechos o sucesos, ocasionados de manera intencional o no intencional en el área laboral, que indica situación de malestar e inconformidad dentro de este espacio, afectando al desarrollo productivo y personal de los profesionales que incursionan en el mismo, produciendo situaciones conflictivas y negativas que alteran la dinámica "normal" de sus actividades diarias. Para algunos investigadores, los factores de riesgos psicosociales son características condicionantes del trabajo derivado de una baja organización laboral que afecta la salud de manera consciente o inconsciente de los involucrados. "El exceso de exigencias psicológicas del trabajo; La falta de influencia y de desarrollo en el trabajo; La falta de apoyo social y de calidad de liderazgo; Las escasas compensaciones del trabajo". Rodríguez (2010), citado por Macías y Cedeño, (2018).

Una vez analizadas las diversas definiciones, se observa que aun cuando para los autores los riesgos psicosociales son características, condiciones, aspectos, interacciones o derivados de deficiencias de diseño, organización, gestión, existe coincidencia en el efecto o resultado que esos riesgos ejercen sobre la salud física y psicológica del trabajador $\mathrm{y}$ por ende en la productividad y desarrollo personal y empresarial.

Aunado a lo expuesto, se presentan las demandas y exigencias del mundo laboral actual, y los riesgos psicosociales suman un componente de incertidumbre, que deviene justamente de la imposibilidad de controlar todos los factores y particularmente el comportamiento de la demanda, lo que se traduciría en un temor frente a la inestabilidad del empleo, ya que el principal factor de ajuste es el despido, y la pérdida del empleo, o el miedo a perderlo, se constituye en un marco de inseguridad, que afecta el colectivo laboral y que genera fuertes presiones sobre las condiciones psicosociales de trabajo, generando cuadros de estrés, riesgos en la salud física y mental y crecientes grados de insatisfacción laboral, que inciden en un ambiente laboral no saludable.

En respuesta a esos riesgos que afectan la salud física y mental de los trabajadores, la OMS (2010) llevó a cabo una investigación dirigida a conocer cómo se pueden mejorar los entornos laborales y establecer una guía que permita a las empresas aplicar las medidas correctas para logarlo, en el informe final publicado participaron un total de 56 expertos de 22 países diferentes y determinaron un total de cuatro planos de influencia en el ámbito del trabajo, entre los que se encuentra el ambiente psicosocial.

\subsection{Ambiente psicosocial como factor del espacio laboral}

En este ambiente se recogen los factores como las actitudes, presiones, prácticas, valores de la empresa, cultura de trabajo y relación entre los diferentes actores de la organización. Los problemas más frecuentes son el estrés emocional y mental de los docentes que puede desencadenar en enfermedades como ansiedad crónica u otras dolencias físicas.

Algunos de los riesgos psicosociales se producen por las siguientes razones: 
Mala organización del trabajo (presiones, poca flexibilidad, falta de gratitud, deficiencias de comunicación...) Cultura institucional (falta de normas y procedimientos, acoso, intimidación, discriminación...) Estilo de gestión (deficiente comunicación entre superiores y empleados...) Temor a la pérdida del empleo debido a los movimientos de personal que realiza la institución educativa y la falta de seguridad que transmite.

\subsection{Factores psicosociales de riesgo}

Son factores probables de daño a la salud, son negativos y pueden afectar tanto a la salud física como a la psicológica. Son factores de estrés que pueden alterar y desequilibrar los recursos y a las capacidades de la persona para manejar y responder al flujo de la actividad derivada del trabajo. Son innumerables y pueden provenir de los múltiples componentes del trabajo: falta de control, muchas horas de trabajo, intensidad del ritmo de trabajo, horarios cambiantes e imprevisibles, mala comunicación organizacional ascendente, horizontal o descendente, ambigüedad o sobrecarga de rol u otros más.

Derivado de lo antes planteado, se consideran factores psicosociales de riesgo: la sobre carga laboral, excesivo trabajo bajo presión, liderazgo con fuerte control o débil control, falta de reconocimiento a la labor, tareas exhaustivas y peligrosas, cambios y reestructuras organizacionales bruscas y sin planificación, no posibilidad de pausas, carga mental alta, entre otros.

Según Uribe (2016) se entiende como factores de riesgo psicosociales en el trabajo a los elementos que rodean el ámbito laboral derivados de fenómenos socioeconómicos, políticos y sociales, cuya presencia o ausencia es susceptible de causar perjuicio en las dimensiones psicofisiológica, cognoscitiva, afectiva y conductual; con impacto integral en la salud, desempeño y calidad de vida de las personas. Partiendo de expuesto por diversos autores, existen fuentes de factores psicosociales que incluyen distintos componentes que pudieran modificarse de acuerdo a la dinámica que impone el contexto de las instituciones educativas. Entre éstas fuentes se consideran:

a. Contenido de la tarea o actividad: ritmo continuo o monótono.

b. Sistema o proceso de trabajo: producción en línea, jornadas extensas o nocturnas.

c. Interacciones sociales: características de las redes e interrelaciones en el ambiente de trabajo que pueden incluir confrontaciones, rivalidades, estigmatizaciones o discriminación, conflictos entre compañeros y superiores, hostigamiento laboral entre otros.

d. La organización: filosofía organizacional, perspectivas, estilo de liderazgo, políticas económicas internas y externas, inseguridad en la permanencia en el empleo, conflictos gremiales, descompensaciones salariales, frecuencia de despidos. e. Ambiente físico: sonidos agudos, olores nauseabundos, hacinamiento, espacios de comunicación sin privacidad.

\subsection{Influencia de la auto perceción del docente en el riego laboral}

Mederos et al (2017), consideran que la forma en que los docentes se perciben está estrechamente vinculada con la objetividad del riesgo laboral. En el ámbito universitario se expresa, según los autores, de forma que "las principales representaciones de los profesores sobre la vida universitaria, se debaten entre elementos de gestión, burocráticos y de la ciencia. La realidad es considerada dentro de ellos pero insuficientemente de acuerdo al rol que debe desempeñar en la vida universitaria. En última instancia constituyen el reflejo de lo que a diario acontece en las universidades estudiadas.

La hiperburocratización y el mercantilismo son dos grandes males de la Universidad que se identifican unánimemente y son absolutamente contrarios a lo propio pues frenan su accionar en lo común. Estos elementos que no son áulicos tienen su expresión en las tres funciones sustantivas de la universidad, y constituyen riesgos laborales, por la sobrecarga laboral que resulta la experiencia universitaria en esas condiciones de trabajo."

\subsection{Características de los factores psicosociales de ries- go erísticas de los factores psicosociales de riesgo}

Según Moreno y Báez(2010) los factores de riesgo psicosocial tienen características propias, entre las que destacan:

1) Se extienden en el espacio y el tiempo. Son características globales de la institución educativa u organización. Incluso otros factores laborales como la carga de trabajo, roles o capacidad de control no tienen un lugar y un momento propios, sino que son elementos del propio puesto de trabajo o la tarea, pero que no se circunscriben a los mismos.

2) Dificultad de objetivación. Uno de los grandes problemas de los factores psicosociales es la dificultad para encontrar unidades de medida objetiva. Por definición (OIT, 1986), se basan en "percepciones y experiencias" y en este caso, atendiendo al enfoque organizacional, a la experiencia intersubjetiva del colectivo organizacional.

3) Afectan a los otros riesgos. El aumento de los factores psicosociales de riesgo, el estrés y la tensión, suelen generar conductas precipitadas, alteradas y no adaptativas que propician los errores, todo tipo de accidentes o incidentes y una mayor vulnerabilidad de la persona. El aumento de los factores psico sociales de riesgo supone habitualmente un aumento de los riesgos de seguridad, de higiene y de ergonomía.

4) Tienen escasa cobertura legal. El desarrollo de la legislación sobre aspectos psicosociales se ha trabajado de modo general relacionada con la prohibición de que 
puedan dañar a la salud, lo que suele ser inefectivo para los directivos y autoridades educativas, para los docentes y para la misma inspección del estado.

5) Están moderados por otros factores. La carga de trabajo, el conflicto de rol, la incertidumbre laboral no suelen tener los mismos efectos sobre toda la población docente de una institución educativa, sino que depende de las variables personales de cada uno de ellos, como el nivel de implicación, la confianza en sí mismo, el optimismo y la motivación de logro

6) Dificultad de intervención. Un clima socio laboral nefasto en la institución educativa afecta a la salud de los docentes, y también a su misma productividad. Lo mismo ocurre con un liderazgo abusivo. Sin embargo, técnicamente la intervención no resulta tan clara y sus efectos a medio y largo plazo tampoco.

\section{Riesgo psicosocial laboral}

Son situaciones que afectan habitualmente de forma importante y grave la salud de los docentes, física, social o mentalmente. Un clima laboral autoritario y desconsiderado es un factor psicosocial de riesgo, pero es también el marco propicio para que aparezcan riesgos psicosociales como el abuso, la violencia y el acoso. En este sentido, la prevención comienza al nivel de los factores psicosociales de riesgo.

No es extraño que los riesgos psicosociales tengan consecuencias graves, que alteren el equilibrio mental de la persona con afectaciones claramente psicopatológicas o psiquiátricas. Un ejemplo claro es el estrés postraumático, prácticamente inexistente en los factores psicosociales de riesgo, pero nada extraño en los riesgos psicosociales como las situaciones de violencia, de acoso laboral o sexual, problemas gastrointestinales (gastritis, úlcera, síndrome de colon irritable, entre otros), cardiovasculares (arritmias, presión arterial alta, infartos al miocardio, etc.), mentales (estrés crónico, burnout, depresión, ansiedad, alcoholismo, delirios, esquizofrenia, etc.)

Los riesgos psicosociales, a diferencia de los factores psicosociales, no son condiciones organizacionales sino hechos, situaciones o estados del organismo con una alta probabilidad de dañar la salud de los docentes de forma importante. Los riesgos psicosociales laborales no son autónomos sino que tienen sus antecedentes en los factores psicosociales de riesgo.

\section{Consideraciones Finales}

Para que exista un entorno laboral saludable en las instituciones educativas, éste debe promover la participación de los docentes, trabajadores y diferentes actores sociales interesados en la realización de acciones conjuntas para controlar, mejorar y mantener la salud y el bienestar de todos los agentes que desempeñan su labor. Un ambiente laboral saludable mejora las condiciones de la productividad, por lo tanto, la calidad de vida del conglomerado de la institución educativa. Se debe promover programas y actividades sobre la conservación de la salud en el lugar de trabajo para evitar riesgos psicosociales, involucrando al docente en la toma de decisiones inherentes a sus tareas laborales.

\section{Referencias Bibliográficas}

Bezanilla, J. M., Carreon, A., Bedolla, E., y Carrilllo, G. (2018). Estudio diagnóstico de factores de riesgo psicosocial en una institucion educativa. Descargado 19/10/2018, de https: / / goo.gl/5 JKAZc

Macías Niemes, I., y Cedeño Barreto, M. (2018). Análisis de los riesgos psicosociales en el área de educación en docentes de la unidad educativa fiscal "tosagua" provincia de Manabí, país Ecuador. 2017. Descargado de https: / / goo.gl/MdAGQp

Mederos Machado, M. C., Balmaseda Espinosa, C. E., y Balmaseda Mederos, M. (2017). Apreciación de los docentes acerca de la vida universitaria. estudio en universidades ecuatorianas (Vol. Núm. 19). Descargado de https://goo.gl/hV6W1U

Moreno Jiménez, B., y Báez León, C. (2010). Factores y riesgos psicosociales, formas, consecuencias, medidas y buenas prácticas. España. Descargado de https: / /goo.gl/RqqQoe

Organización Mundial de la Salud. (2010). Entornos saludables: Fundamentos y modelo de la oms. contextualización, prácticas y literatura de soporte. Ginebra, Suiza.

Organización Panamericana de la Salud. (2015). Plan de acción sobre la salud de los trabajadores 2015 2015.

Uribe Prado, J. (2016). Psicología del trabajo: Un entorno de factores psicosociales saludables para la productividad. Editorial El Manual Moderno. Descargado de https://goo.gl/MJzrNU

Zimmermann, M. (2016, 06). La Estrategia Española de Seguridad y Salud en el Trabajo 2015-2020. Archivos de Prevención de Riesgos Laborales, 19, 83 - 85. Descargado de https://goo.gl/YxiEKb doi: 10.12961/aprl.2016.19.02.1

Recibido: 8 de agosto de 2018

Aceptado: 15 de octubre de 2018 
